\title{
Survey Methods for Assessment of Citrus Tristeza Virus Incidence
}

\author{
G. Hughes and T. R. Gottwald
}

First author: Institute of Ecology and Resource Management, University of Edinburgh, West Mains Road, Edinburgh EH9 3JG, Scotland, United Kingdom; and second author: USDA Agricultural Research Service, Horticultural Research Laboratory, 2120 Camden Road, Orlando, FL 32803.

Accepted for publication 30 March 1998.

ABSTRACT

\begin{abstract}
Hughes, G., and Gottwald, T. R. 1998. Survey methods for assessment of citrus tristeza virus incidence. Phytopathology 88:715-723.

The assessment of citrus tristeza virus incidence by sampling involves laboratory testing by enzyme-linked immunosorbent assay of leaf material collected in the field. Using field data and computer simulation, methods of field data collection were compared. One method was similar to that used by the Central California Tristeza Eradication Agency, in which 4 to $6 \%$ of the trees in a planting block are sampled and material from each tree sampled is assayed separately. This meth-
\end{abstract}

od was compared with an alternative method in which about $25 \%$ of the trees in a block are sampled, and material from groups of four trees is bulked and assayed together. Our comparative study indicated that the latter method results in increased accuracy and precision of estimates of citrus tristeza virus incidence without increasing unduly the number of laboratory assays required.

Additional keywords: Aphis gossypii, cluster sampling, group testing, hierarchical sampling, spatial pattern, Toxoptera citricida.
In the United States, an active citrus tristeza virus (CTV) eradication program is currently underway in the Central Valley of California. An integral part of such an eradication program is a sampling scheme by which virus-infected trees are detected and the incidence of virus infection estimated. The Central California Tristeza Eradication Agency (CCTEA), established in 1963, has been using a systematic sampling scheme to conduct an extensive survey of the incidence of CTV in commercial citrus in the Central Valley. Laboratory-based enzyme-linked immunosorbent assay (ELISA) of the material sampled in the field is used to classify individual trees as either CTV-positive or CTV-negative. Thus, incidence (the proportion of CTV-positive trees) can be estimated, as a basis for disease management decision making.

Sampling schemes for use in very large citrus-growing areas must be practical in terms of time, labor, and expense. For a CTV eradication program, a threshold of CTV incidence is established that, if exceeded, invokes a tree-by-tree census to determine the location of individual CTV-positive trees. These are subsequently removed. Thus, the key to the success of an eradication program is a sampling scheme that is capable of detecting CTV infection at low incidence and doing so relatively quickly and economically.

In the CCTEA sampling scheme, every fifth tree in every fifth row is selected for sampling. In this paper, the use of an alternative sampling scheme was investigated. In the alternative scheme, material is collected and assayed from groups of four trees, without distinguishing the individual trees. CTV incidence at the scale of the individual tree can be calculated by means of an appropriate formula (15). Estimates of CTV incidence made in this way were compared with estimates made from data obtained by sampling

Corresponding author: G. Hughes; E-mail address: ghughes@srv0.bio.ed.ac.uk

Publication no. P-1998-0518-03R

This article is in the public domain and not copyrightable. It may be freely reprinted with customary crediting of the source. The American Phytopathological Society, 1998. directly at the individual tree level, as in the CCTEA sampling scheme, using field data and computer simulation.

The usual motivation for testing groups of material collected by sampling in the field is to reduce the number of tests required and so reduce the cost of a survey or an experiment. However, in the context of the current study of CTV incidence, testing of material in groups provided a means of increasing the proportion of the population sampled without increasing to the same extent the number of tests required. This would be of practical importance if the accuracy and precision of estimates of CTV incidence so obtained represented an improvement compared with those provided by the CCTEA sampling scheme. Reduction of the number of tests was, therefore, not a concern in this case.

The objectives of this study were to evaluate the accuracy and precision of estimates of incidence of CTV infection provided by the CCTEA sampling scheme and to develop and evaluate an alternative methodology. The need for accuracy and precision of estimates at low (i.e., up to $10 \%$ ) incidence was borne in mind, as was the requirement for economical use of resources. The study raised a number of important methodological issues including characterization of the spatial pattern of infected plants, estimation of CTV incidence at the individual tree scale after testing groups of trees, determination of the appropriate number and size of groups of trees, and validation of sampling schemes.

\section{MATERIALS AND METHODS}

Sampling individual trees. Mainly for reasons of practicality, the CCTEA sampling scheme is systematic, every fifth tree in every fifth row being selected for sampling. Procedures for systematic sampling and their advantages and disadvantages are discussed by Sampford (25). In order for the mean of a systematic sample to provide an unbiased estimate of the population mean, some element of randomization must be incorporated into the sampling procedure. In the case of the CCTEA scheme, this may be achieved by selecting the initial tree to be sampled at random from among any of the first five trees in the first five rows in the block in 
question and subsequently selecting every fifth tree in every fifth row systematically. In practice, selection of a random starting position has not been routinely used by the CCTEA. Rather, the first tree in the first row has been used as the initial starting position. However, in the simulation study of sampling methods (described below), random starting positions were implemented by means of the RND function in a Microsoft Excel spreadsheet macro (Microsoft Corp., Redmond, WA).

In most cases, a systematic sample ought not to be analyzed simply as if it were a random sample (25). An exception to this is if the population to be sampled is randomly ordered. An analysis of the pattern of CTV incidence in the field is, therefore, a precursor to our investigation of sampling strategies and the analysis of the data that they provide.

Sampling groups of trees. First, consider a recent example of 'group testing' applied to the problem of estimating virus incidence $(12,24)$. The study involved assessment of the level of infection of cut-flower 'Sim' carnations by a number of viruses. Each assessment involved taking a systematic sample of 200 leaves, one from each plant on a grid. After picking, the leaves were combined into a single lot of 200 and transported to the laboratory for ELISA testing. A question that arises in such situations is whether to estimate the level of virus incidence by testing each of the leaves separately or after combining the leaves into groups. If the latter course of action is chosen, the question of what size of group is to be used also arises. Discussions of the statistical justification for group testing can be found in the literature cited by Hepworth (12).

Assuming that a group testing approach is adopted, the procedure for estimating incidence at the scale of the individual can be summarized as follows. Calculations relating to the lower of the two scales (in the carnation virus example, the leaf scale) are denoted by the subscript 'low', while those relating to the higher of the two scales (the group scale) are denoted by the subscript ' $h i g h$ '. The mean proportion of infected leaves per group (i.e., observed mean incidence measured at the leaf scale) is denoted as $\hat{p}_{\text {low }}$. When the group size is constant,

$$
\hat{p}_{\text {low }}=\left(\sum_{i} \hat{p}_{\text {low }, i}\right) / N \quad ; \quad i=1,2, \ldots, N
$$

in which $\hat{p}_{\text {low }, i}$ is the proportion of leaves infected in the $i$ th group, and there are $N$ groups. The proportion of groups with at least one infected leaf (i.e., observed mean incidence measured at the higher of the two scales) is denoted as $\hat{p}_{\text {high }}$. The proportions $\hat{p}_{\text {low }}$ and $\hat{p}_{\text {high }}$ are estimates of, respectively, $p_{\text {low }}$ (the unobserved probability that a leaf is infected in a given group) and $p_{\text {high }}$ (the unobserved probability that a group has at least one infected leaf).

In cases like the carnation virus example, in which leaves are essentially assigned to groups at random, the binomial distribution may be assumed to provide an appropriate description of the frequency of infected leaves per group. The zero term of the distribution (the probability that a group has no infected leaves) is given by $\left(1-p_{\text {low }}\right)^{n}$ ( $n$ being the number of leaves per group), so $p_{\text {high }}=1-$ $\left(1-p_{\text {low }}\right)^{n}$. By straightforward rearrangement, $p_{\text {low }}=1-(1-$ $\left.p_{\text {high }}\right)^{1 / n}$, and the mean incidence at the lower scale may be estimated from observations made at the higher scale,

$$
\widetilde{p}_{\text {low }}=1-\left(1-\hat{p}_{\text {high }}\right)^{1 / n}
$$

in which a tilde refers to an estimate that has been made from observations at a spatial scale other than the one denoted by its subscript.

The carnation virus example is typical of group testing in that, after transportation to the laboratory, leaves could be regarded as an unrestricted random sample from plants in the field. In group testing, locations of plants from which individual leaves were taken are not recorded. The problem is solely one of estimating inci- dence at the individual scale from observations at the group scale. However, in the case of CTV assessments, information about the spatial pattern of CTV infection may be of epidemiological significance (10).

Even if intensive mapping is not carried out, information about the spatial pattern of CTV infection can be obtained by cluster sampling in the field (14). Such a procedure involves the assessment of individual trees in sampling units ('quadrats') comprising a number of adjacent trees. The theory relating to group testing is relevant if the material from each quadrat is pooled and tested together. But, because the CTV status of a tree may not be independent of the CTV status of its neighbors, use of equation 2 for the estimation of $\tilde{p}_{\text {low }}$ requires more explicit justification than in cases in which material collected in the field is randomly allocated to groups in the laboratory. Such justification would come in the form of an analysis of the spatial pattern of field data for CTV incidence, showing that the binomial distribution provided an appropriate description of the frequency distribution of infected trees per quadrat. The spatial analysis mentioned above as a precursor to the discussion of sampling strategies is, therefore, also required in this context.

In many respects, a procedure that involves cluster sampling in the field, with virus assessments made at the level of the cluster rather than at the level of individuals within clusters, is similar to so-called binomial sampling $(16,22)$. In the context of pathology, binomial sampling is based on relationships between disease incidence and disease severity, as discussed by Seem (26). The technique for making estimates of disease severity from incidence data collected in the field relies on the relationship between the zero term of a probability distribution of counts and the mean of the distribution $(3,6)$. To emphasize the similarity between binomial sampling and cluster sampling with assessment at the level of the cluster (rather than at the level of individuals within clusters), we use the term 'hierarchical sampling' for our procedure.

Although hierarchical sampling borrows its theoretical justification entirely from group testing, it differs from conventional group testing (and resembles binomial sampling) in that it involves a hierarchy of data with an explicit spatial component. Hierarchical sampling also differs from conventional group testing in the matter of choice of group size. In group testing, much attention has been devoted to the choice of an appropriate group size $(12,28,29)$. An experimenter has great flexibility in making this choice, since material is combined into groups in the laboratory after sampling has taken place in the field. In hierarchical sampling, the choice of sampling unit (i.e., the number of individuals per group, cluster, or quadrat) must, in addition to acknowledging statistical requirements, take into account practical aspects of field sampling. In the current study, a quadrat size of four trees, arranged two rows by two trees along rows, was chosen. The practical and statistical implications of this choice are discussed later. A third point of detail in which the hierarchical sampling scheme used in the current study differs from conventional group testing concerns the retesting of infected groups. If the only concern is the estimation of $\tilde{p}_{\text {low }}$, retesting is of little value (5). However, in the case of CTV, trees that can be identified by testing as infected may subsequently be removed. Thus, if the absolute spatial locations of groups in the field are carefully recorded, retesting of individuals in infected groups may provide information of use in disease management.

There is another issue to be addressed in relation to testing of material in groups. The laboratory assay must be able to detect a single infected individual in a group. An experiment was conducted to test the ability of the current ELISA method to detect a single CTV-infected tree among a group of trees. A dilution series consisting of 8:0, 7:1, 6:2, 5:3, 4:4, 3:5, 2:6, 1:7, and 0:8 (control) CTV-positive/CTV-negative leaf petioles was tested against a dilution series of 1:20, 1:40, 1:80, 1:160, 1:320, and 1:640 CTV-antiserum/buffer. The CTV-antiserum consisted of a mixture of two 
monoclonal antibodies, 11B1 and 3E10, that, in combination, act as a universal CTV probe capable of detecting all known CTV isolates. A sample was considered CTV-positive if the optical density (at $405 \mathrm{~nm}$ ) of the reaction was greater than twice that of the control. The standard double-antibody sandwich indirect ELISA protocol was used (7).

Field data from previous studies. Data from 36 rectangular blocks of citrus from surveys made during 1992 and 1993 in California were available from CCTEA records. These blocks varied in size between 472 and 3,000 trees. The location and CTV status of each tree had been recorded in the form of a map. CTV status of individual trees had been determined by ELISA as either CTVnegative or CTV-positive. The range of CTV incidence recorded was 0.4 to $19 \%$.

The map of each block was divided into $N$ quadrats (in this case, $N$ varies from block to block) of $n=$ four trees, arranged two rows by two trees along rows. The frequency distribution of CTVpositive trees per quadrat was then compiled for each block. The empirical (observed) variance of each of these distributions $\left(v_{\text {low }}\right)$ was estimated from

$$
\hat{v}_{\text {low }}=\left[\sum_{i}\left(\hat{p}_{\text {low }, i}\right)^{2}-\left(\sum_{i} \hat{p}_{\text {low }, i}\right)^{2} / N\right] /(N-1)
$$

and the corresponding theoretical (binomial) variance $\left(v_{l o w, b i n}\right)$ was estimated from

$$
\hat{v}_{\text {low }, \text { bin }}=\hat{p}_{\text {low }} \cdot\left(1-\hat{p}_{\text {low }}\right) / n
$$

A linear relationship, on logarithmic axes, between $\hat{v}_{\text {low }}$ and $\hat{v}_{\text {low,bin }}$ can be interpreted as an indicator of the spatial pattern of incidence $(13,19)$. A previous example of such an analysis for CTV incidence used data from eastern Spain (9) to show that the pattern of CTV-positive trees at the within-quadrat scale was indistinguishable from random (15). In both the Spanish case and that of the data set from California, the main vector of CTV was the aphid Aphis gossypii.

The maps from 12 of the 36 blocks (covering the whole observed range of incidence) were also used as a basis for illustrating some characteristics of the CCTEA sampling scheme. For each selected map, all 25 possible estimates of incidence were calculated. This was achieved by systematically sampling every fifth tree in every fifth row, starting the sampling in turn from each of the 25 possible starting positions on each map.

Simulated data. Simulated CTV-incidence data were generated using a Microsoft Excel spreadsheet macro, CTVSamplingSimulator (T. R. Gottwald, unpublished data). This simulator can generate arrays (with user-defined numbers of rows and columns) in which a user-defined percentage of individual cells is designated ' 1 ' (CTV-positive) at random, the remaining cells being designated '0' (CTV-negative). We will refer to these simulated arrays as blocks and cells within them as trees, making clear either explicitly or by context the distinction between simulated data and field data.

To validate the simulator, blocks of 400 trees, arranged 20 rows by 20 columns, were generated. Ten blocks were generated at each of following incidences: $0.5,1.0,1.5,2.0,2.5,3.0,3.5,4.0,4.5$, 5.0, 5.5, 6.0, 6.5, 7.0, 7.5, 8.0, 8.5, 9.0, 9.5 and 10.0\% CTV-positive trees. The blocks were divided into quadrats of $n=$ four trees, arranged two by two, and the number of quadrats in each block with $0,1,2,3$, or 4 CTV-positive trees was calculated (these operations are available as user-requested functions of the simulator). Thus, the frequency distribution of CTV-positive trees per quadrat was compiled for each simulated block. Both the observed variance and the corresponding binomial variance of each of these frequency distributions were calculated, using equations 3 and 4, respectively, in preparation for analysis by the same method as was used for the field data.

Simulation study of sampling strategies. A number of sampling schemes were investigated by simulation. Here, a compari- son of four different schemes is reported. The sampling schemes reported are as follows.

A. Sampling individual trees: Every fifth tree in every fifth row. The initial tree was selected at random from among the first five trees in each of the first five rows, and then every fifth tree in every fifth row, systematically, after that. One out of every twenty-five trees, or $4 \%$ of the population, was sampled. This is referred to as the CCTEA scheme.

B. Sampling individual trees: Every fourth tree in every fourth row. The initial tree was selected at random from among the first four trees in each of the first four rows, and then every fourth tree in every fourth row, systematically, after that. One out of every sixteen trees, or $6.25 \%$ of the population, was sampled.

C. Sampling individual trees: Every second tree in every second row. The initial tree was selected at random from among the first two trees in each of the first two rows, and then every second tree in every second row, systematically, after that. One out of every four trees, or $25 \%$ of the population, was sampled.

D. Sampling groups of trees. A 20 by 20 block was considered to be 100 groups of four individual trees, each group arranged two by two. One out of the first four groups was selected at random, and then every fourth group, systematically, after that. Four out of every sixteen trees were sampled $(25 \%$ of the population), but only CTV incidence at the group level (either no CTV-positive individuals or at least one CTV-positive individual) was recorded.

Scheme A is reported in order to be able to make comparisons between the CCTEA scheme and others. Scheme D is the proposed hierarchical sampling scheme. Scheme B requires the same number of tests as $\mathrm{D}$, but involves sampling individual trees. Scheme C involves sampling the same percentage of the population as $\mathrm{D}$, but as individuals rather than in groups, so it requires four times the number of tests as D.

Each scheme was the subject of a simulation experiment based on the generation of blocks, 20 rows by 20 columns in size, in which the positions of CTV-positive trees were allocated at random. For each of the four schemes, 100 replicate blocks were simulated at each of the following incidences: 1, 2, 3, 4, 5, 6, 7, 8, 9, and $10 \%$ CTV-positive trees. For sampling schemes A, B, and C, $\hat{p}_{\text {low }}$ was calculated for each replication using equation 1 . For sampling scheme $\mathrm{D}, \widetilde{p}_{\text {low }}$ was calculated for each replication using equation 2 .

Operating characteristic curves. So-called 'operating characteristic (OC) curves' are widely used in economic entomology to describe the performance of sampling schemes, particularly those based on sequential sampling $(2,16,21)$. Although CTV sampling schemes are not sequential (because the virus-detection procedures are, of necessity, laboratory-based), the same concept is useful here. First, an appropriate decision threshold is defined. Here, for example, $10 \%$ CTV-positive trees was adopted as the threshold $\left(t_{\text {low }}=0.1\right)$. Then, for sampling schemes $\mathrm{A}, \mathrm{B}$, and $\mathrm{C}, T_{\text {low }}$ is defined as the largest integer less than or equal to $t_{\text {low }} \cdot N$. $T_{\text {low }}$ is, thus, the largest number of CTV-positive trees, out of a total of $N$ trees, that can be observed without $t_{\text {low }}$ being exceeded. The OC curve then shows the probability that the observed number of CTV-positive trees $(X)$ is less than or equal to $T_{\text {low }}$ :

$$
P\left(X \leq T_{\text {low }}\right)=\sum_{x=0}^{T_{\text {low }}} P(X=x)
$$

in which $P(X=x)$ is based on the binomial distribution. This represents the probability of a decision (correct or otherwise) that the incidence of CTV-positive trees is less than or equal to the adopted threshold of $10 \%$ for any actual value of incidence.

For sampling scheme $\mathrm{D}$, in which the quadrat ( $n=$ four trees) rather than the individual tree is the sampling unit, the corresponding threshold is $t_{\text {high }}=1-\left(1-t_{\text {low }}\right)^{n}$. Then, $T_{\text {high }}$ is defined as the largest integer less than or equal to $t_{\text {high }} \cdot N . T_{\text {high }}$ is, thus, the largest 
number of quadrats with at least one CTV-positive tree, out of a total of $N$ quadrats, that can be observed without $t_{\text {high }}$ being exceeded (and so, also, the largest number of quadrats with at least one CTV-positive tree that can be observed without $t_{\text {low }}$ being exceeded). The OC curve then shows the probability that the observed number of quadrats with at least one CTV-positive tree $(X)$ is less than or equal to $T_{\text {high }}$ :

$$
P\left(X \leq T_{\text {high }}\right)=\sum_{x=0}^{T_{\text {high }}} P(X=x)
$$

in which, as in equation $5, P(X=x)$ is based on the binomial distribution. As with equation 5 , this represents the probability of a decision (correct or otherwise) that the incidence of CTVpositive trees is less than or equal to the adopted threshold (at the individual tree scale) of $10 \%$ for any actual value of incidence at that scale.

Realized OC curves for the sampling schemes were obtained by inspection of the output from the simulation study of sampling strategies. At each actual CTV incidence, the number of occurrences of estimated CTV incidence less than or equal to the adopted threshold of 0.1 (10\% CTV-positive trees) was simply counted and expressed as a proportion of the 100 simulations at each actual incidence.

Field data from concurrent studies. With the assistance of CCTEA personnel, data were collected in December 1996 from 10 commercial citrus blocks in California by sampling all trees in each block individually, in order to determine the actual CTV incidence via ELISA. At the same time, sampling schemes A and D were used (in the field, not on the map prepared from the census results) to estimate CTV incidence. Using these data, a small-scale

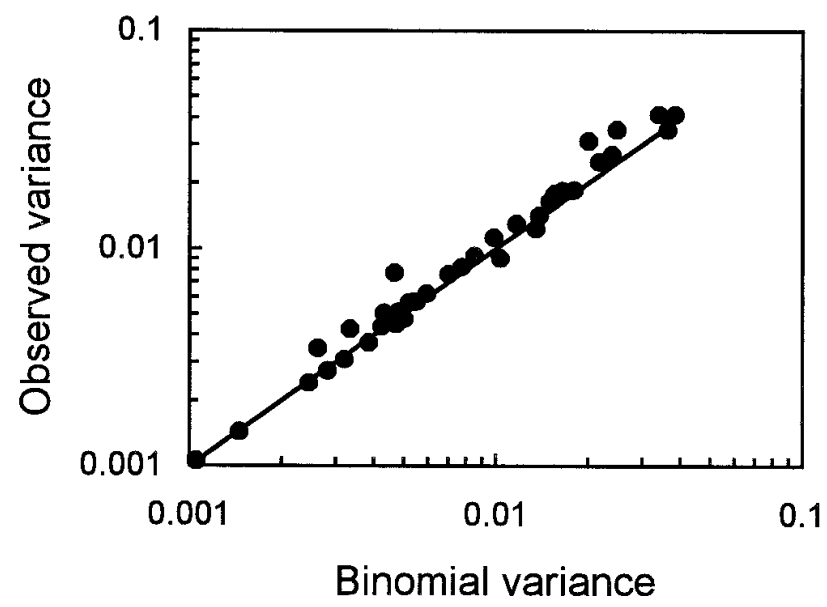

Fig. 1. The relationship between the observed and the theoretical binomial (random) variances of incidence of citrus tristeza virus (CTV) at the tree scale (note the use of logarithmic scales on both axes). Each data point represents a CTV assessment in a block of citrus in the Central Valley of California. The solid line represents the binomial line (i.e., observed variance $=$ binomial variance). comparison of the performance of sampling schemes A and D in the field against known standards was made.

\section{RESULTS}

Spatial pattern analysis. For the 36 field plots for which data were available, the relationship between the observed variance ( $\hat{v}_{\text {low }}$, calculated from equation 3 ) and the corresponding binomial variance $\left(\hat{v}_{\text {low,bin }}\right.$, calculated from equation 4 ) was very close to the 'binomial line' (Fig. 1). The slope and intercept of the least squares linear regression line fitted to the data (plotted on logarithmic axes) were, respectively, 1.03 (SE [standard error] $=0.026$ ) and $0.10(\mathrm{SE}=0.054)$. There is, thus, no suggestion that the data can be distinguished from the 'binomial line', and this is taken as evidence that the binomial distribution provides an appropriate description of the frequency distribution of CTV-positive trees per quadrat. That is to say, the pattern of CTV-positive trees may be taken as random at the within-quadrat scale (19). This means that the formula for the calculation of sample statistics may be calculated using a formula appropriate for unrestricted random samples, even though sampling is actually systematic. Note that the analysis carried out here provides a summary of the characteristics of a small-scale pattern, useful in the context of sampling, but that it does not tell us about the dispersal processes that led to the observed pattern. Other methods are available when that is the objective (8).

The data on which Figure 2 is based were generated using $C T V$ SamplingSimulator. At intervals of $0.5 \%$ between 0.5 and $10 \%$ CTV incidence, 10 blocks of 400 trees, arranged 20 rows by 20

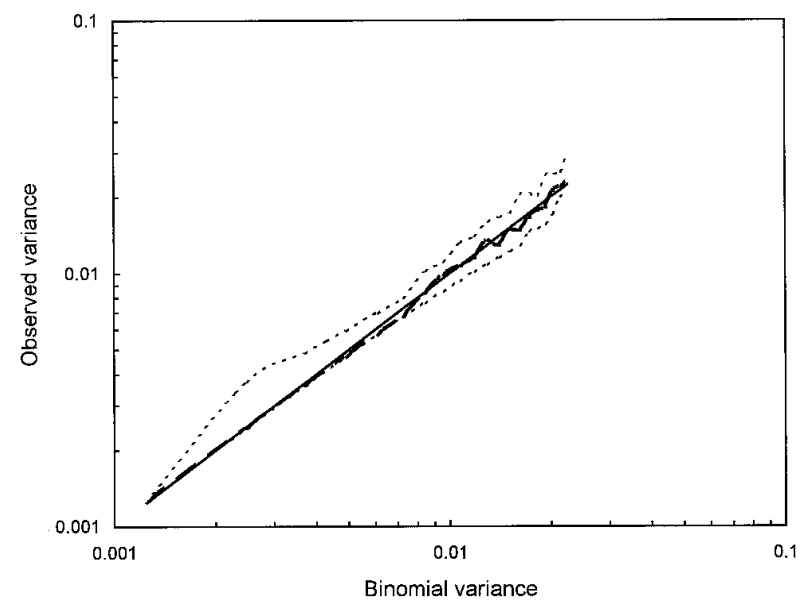

Fig. 2. The relationship between the observed and the theoretical binomial (random) variances of incidence for 200 simulations of citrus tristeza virus (CTV) incidence generated by CTVSamplingSimulator (note the use of logarithmic scales on both axes). The dotted interval (- - ) shows the range covering all simulations (there were 10 at each incidence: $0.005,0.01,0.015, \ldots$, $0.1)$. The dashed line $\left(-\_-\right)$represents the median value of observed variance at each binomial variance. The solid line represents the binomial line (i.e., observed variance $=$ binomial variance).

TABLE 1. Demonstration of enzyme-linked immunosorbent assay ability to detect citrus tristeza virus (CTV)-positive tissue in mixtures of CTV-positive and CTV-negative leaf petioles

\begin{tabular}{|c|c|c|c|c|c|c|c|c|c|}
\hline \multirow[b]{2}{*}{ Antiserum $^{\mathrm{b}}$ dilution } & \multicolumn{9}{|c|}{ CTV-positive/CTV-negative leaf petioles ${ }^{\mathrm{a}}$} \\
\hline & $8: 0$ & $7: 1$ & $6: 2$ & $5: 3$ & $4: 4$ & $3: 5$ & $2: 6$ & $1: 7$ & $0: 8$ \\
\hline $1: 20$ & 1.107 & 1.036 & 0.859 & 0.936 & 0.928 & 0.965 & 0.829 & 0.682 & 0.042 \\
\hline $1: 40$ & 0.959 & 1.023 & 1.064 & 0.845 & 0.797 & 0.905 & 0.749 & 0.633 & 0.020 \\
\hline $1: 80$ & 1.249 & 1.046 & 1.296 & 0.810 & 0.947 & 0.879 & 0.620 & 0.528 & 0.016 \\
\hline $1: 160$ & 1.375 & 1.203 & 1.335 & 1.087 & 0.884 & 1.318 & 0.507 & 0.243 & 0.051 \\
\hline $1: 320$ & 1.036 & 1.714 & 1.245 & 1.070 & 0.728 & 0.639 & 0.359 & 0.155 & 0.029 \\
\hline $1: 640$ & 0.938 & 0.660 & 1.076 & 0.789 & 0.486 & 0.412 & 0.212 & 0.099 & 0.025 \\
\hline
\end{tabular}

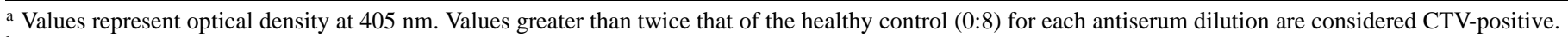

b Antiserum consisted of a mixture of two monoclonal antibodies, 11B1 and 3E10, that in combination act as a universal probe for CTV detection. 
columns, were generated and divided into quadrats in the same way as for the field data. The figure shows the median observed variance and the interval within which all of the simulated values fell, plotted against the corresponding binomial variance. Since

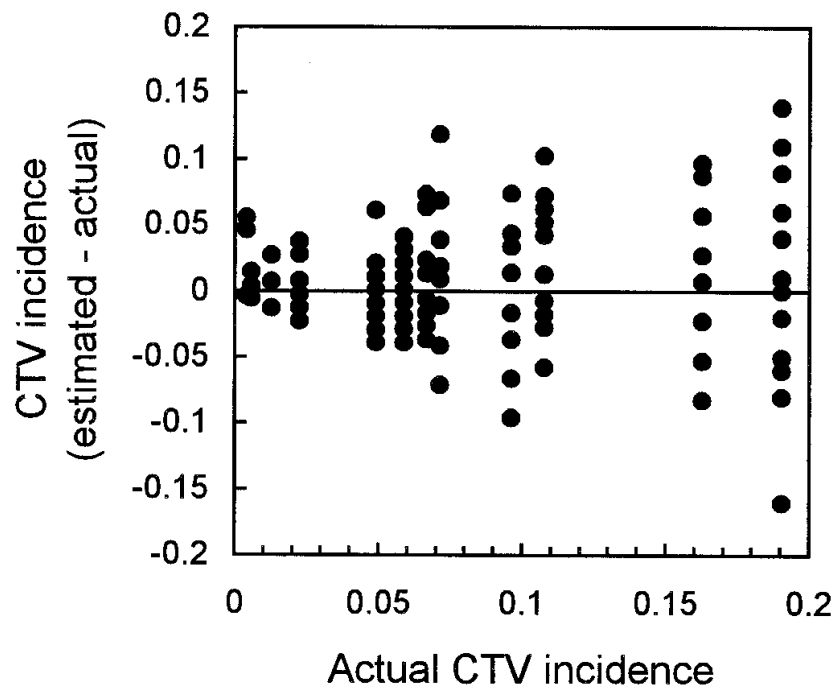

Fig. 3. For each of 12 maps of citrus tristeza virus (CTV) incidence, the Central California Tristeza Eradication Agency sampling scheme was used, beginning in turn in each of the 25 possible starting locations, to calculate all 25 possible estimates of incidence. The data points show differences between estimated and actual incidence (several data points may overlap at each actual CTV incidence).

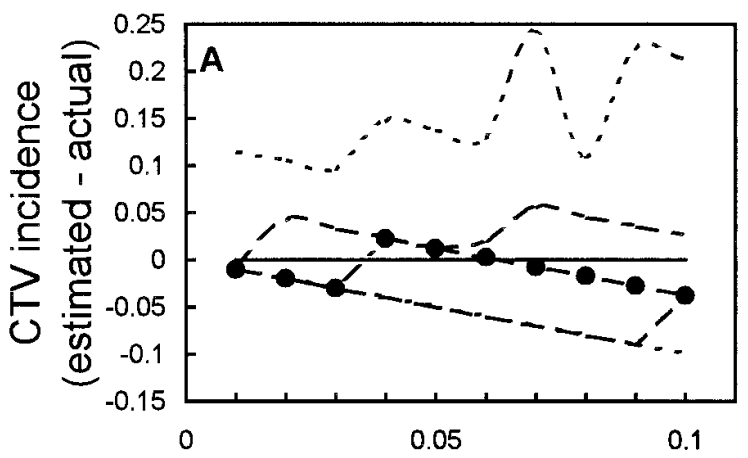

Actual CTV incidence

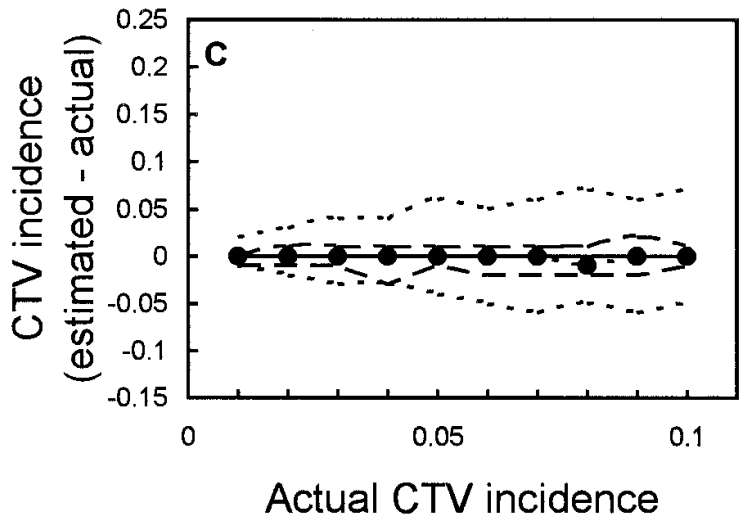

this analysis of the simulated data resembles closely the analysis of the field data shown in Figure 1, we conclude that the simulator provides a valid basis for the study of sampling schemes for CTV incidence in cases in which the pattern of CTV-positive trees can be taken as random at the within-quadrat scale.

ELISA sensitivity. The ability of ELISA to detect various mixtures of CTV-positive and CTV-negative tissue is shown in Table 1. The test demonstrated that the assay could detect a single CTV-infected petiole when mixed with seven healthy petioles. The antiserum dilution series further demonstrates that such discrimination is possible even at a very low antiserum concentration, suggesting that CTV detection may be possible even when the ratio of CTV-positive/CTV-negative material is smaller than $1: 7$.

Field data from previous studies. Figure 3 shows the results of taking all 25 possible systematic samples of every fifth tree in every fifth row from each of 12 maps available from previous studies of CTV incidence. The range of estimates of incidence that may be made from a map is indicated by the vertical scatter of points at each actual CTV incidence. At around actual CTV incidences of 0.1 , for example, estimated CTV incidences as low as 0 and as high as 0.2 were obtained. It may be concluded that the accuracy offered by this sampling scheme is insufficient for the purposes for which it is required.

Simulated data. Figure 4 shows the results of the simulation study of sampling strategies. For each sampling scheme and level of CTV incidence, the difference between the CTV incidence estimated by sampling and the actual incidence was calculated separately for each of 100 replicate simulations. The outer interval (dotted lines) in each part of Figure 4 shows the range of values
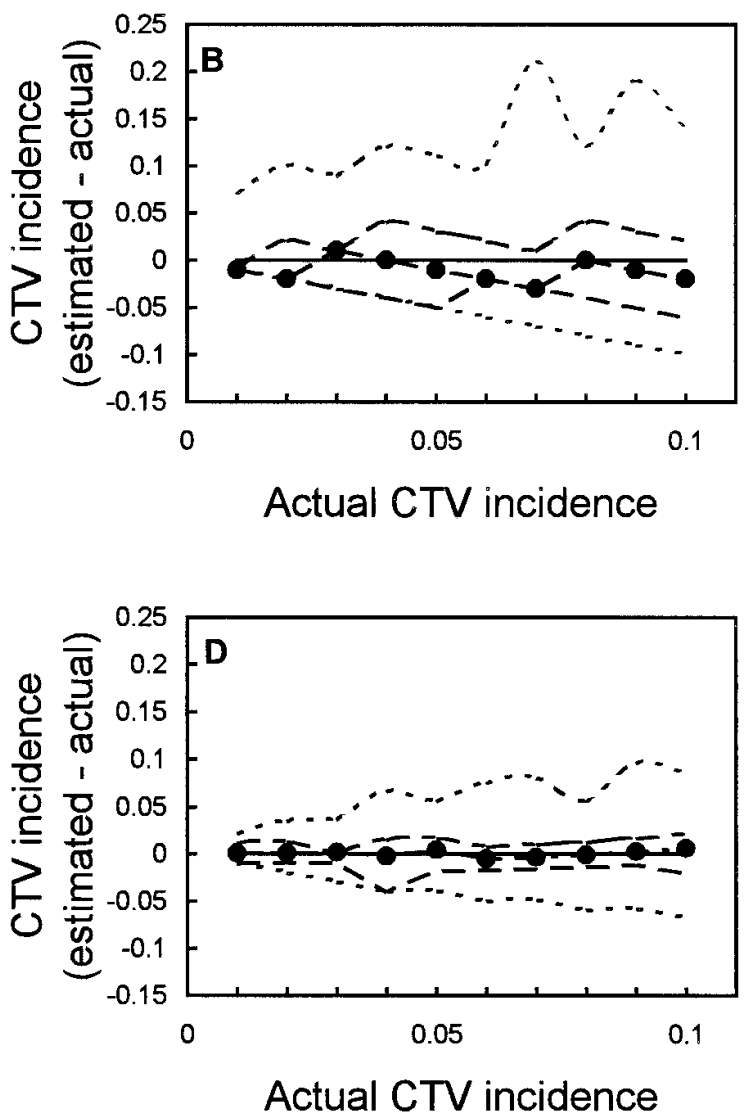

Fig. 4. The results of the simulation study of sampling strategies. The differences are shown between the observed and the actual citrus tristeza virus (CTV) incidence over a range of values of actual CTV incidence. The dotted interval (- - ) shows the range covering all simulations (there were 100 at each incidence: $0.01,0.02,0.03, \ldots, 0.10)$. The dashed interval $(---)$ shows the range covering the 25 th to the 75 th percentiles (i.e., the range covering half the simulations). The dashed line joining the data points (- - - ) shows the median value of the simulations. The horizontal solid line represents zero difference between the observed and the actual CTV incidences. A, Sampling individual trees: every fifth tree in every fifth row. B, Sampling individual trees: every fourth tree in every fourth row. C, Sampling individual trees: every second tree in every second row. D, Sampling groups of trees: every fourth group of four trees. 
obtained for the difference between observed and actual incidence. The inner interval (dashed lines) shows the range between the 25th and 75th percentiles, i.e., the range covering half of the values obtained for the difference between observed and actual incidence. The data points in each part of Figure 4 show the median difference between observed and actual incidence. In Figure 4, more accurate estimates are indicated by relatively small differences between observed and actual incidence. More precise estimates are indicated by relatively narrow intervals.

It is clear that sampling scheme A (Fig. 4A) is the poorest in terms of both accuracy and precision. Note in particular that the lower limit of the range coincides with the 25th percentile up to and including an actual CTV incidence of $9 \%$. That is to say, even when the actual incidence is $9 \%$, at least a quarter of the simulations found no CTV-positive trees among those sampled. Any improvement offered by sampling scheme B (Fig. 4B) is marginal. Sampling schemes C (Fig. 4C) and D (Fig. 4D) offer improvements over schemes A and B in terms of both accuracy and precision. The levels of accuracy and precision achieved by schemes $\mathrm{C}$ and $\mathrm{D}$, both of which involve sampling $25 \%$ of the population, are similar. However, since scheme $\mathrm{C}$ involves testing individuals and scheme D involves testing of groups of four, scheme D achieves the improvements in accuracy and precision with one quarter of the number of laboratory tests required for scheme C. Indeed, scheme D requires only the same number of tests as scheme B, but offers both better accuracy and better precision for the same level of effort.

OC curves. Typically, values on an OC curve are near 1 when the actual incidence is much less than the value of incidence defined as the decision threshold, near 0.5 when the actual incidence is near the decision threshold, and near 0 when the actual incidence is much larger than the decision threshold (2). Figure 5 shows these characteristics for all four sampling schemes discussed here, calculated from either equation 5 (sampling schemes A, B, and C) or equation 6 (sampling scheme D). Up to and including the adopted threshold of 0.1 (10\% CTV-positive trees), the OC curves show the true negative proportion (TNP), representing the probability of deciding that a sampled block has an incidence of less than or equal to the threshold, given that the actual incidence is less than or equal to the threshold. Above the adopted threshold of 0.1 (10\% CTV-positive trees), the OC curves show the false negative proportion (FNP), representing the probability of deciding that a sampled block has an incidence of less than or equal to the threshold, given that the actual incidence is greater than the threshold. Sampling schemes C and D are clearly superior to sampling

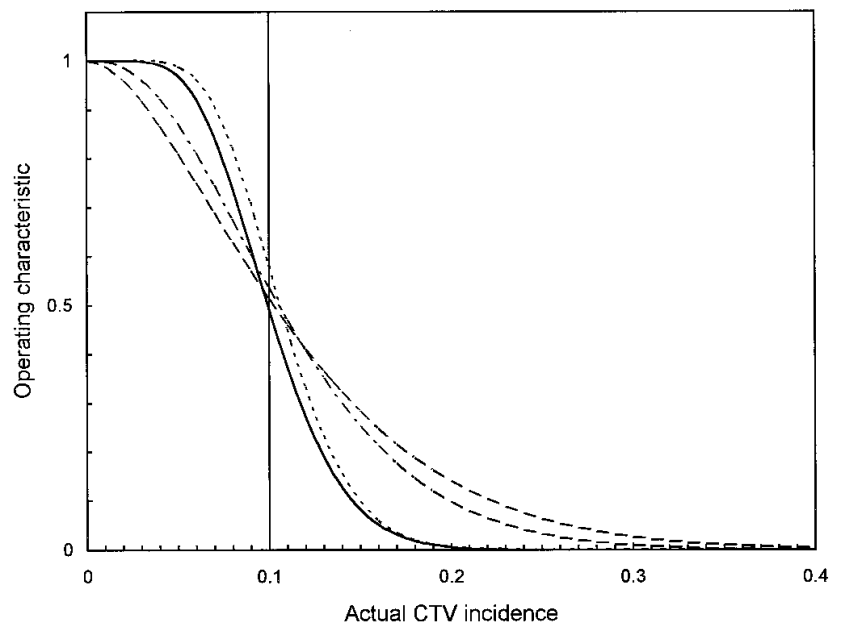

Fig. 5. Theoretical operating characteristic curves for each of the sampling schemes discussed in the current study: Scheme A, - - ; scheme B, - - - - ; scheme $\mathrm{C},---;$ and scheme $\mathrm{D},-$ The vertical line at actual citrus tristeza virus incidence $=0.1$ represents the adopted decision threshold. schemes A and B, providing both higher TNPs and lower FNPs. Sampling scheme C provides slightly higher TNPs than does scheme D, but also slightly higher FNPs. However, as mentioned previously, the true comparison of schemes $\mathrm{C}$ and $\mathrm{D}$ must take into account that scheme $\mathrm{D}$ requires only one quarter of the laboratory costs of scheme $\mathrm{C}$.

We note, in passing, that the OC curve for scheme D applies no matter how the group of four trees is arranged (obviously, since no shape parameter has been introduced). Thus, if it were deemed preferable to collect material from a group of four trees arranged along a single row, such a scheme would have the same characteristics as scheme D (in which the four trees are arranged two by two). That is to say, the spatial arrangement of the group is purely a matter of convenience. It follows that, even if no increase at all can be accommodated in the number of laboratory tests to be carried out, the following sampling scheme is a possible alternative to the CCTEA scheme. A 20 by 20 block is considered to be 80 groups of five individual trees, each group arranged as five trees along a single row. One out of the first five groups is selected at random, and then every fifth group systematically after that. Five out of every twenty-five trees are sampled (20\% of the population), but only the CTV incidence at the group level (either no CTV-positive trees or at least one CTV-positive tree) is recorded.

Figure 6 shows the realized OC curves from the simulation study of sampling strategies. Since all the simulations were carried out in the range up to and including $10 \%$ incidence, Figure 6 shows how the realized TNP changes with actual CTV incidence for each sampling scheme. The resulting OC curves are similar to the corresponding range of the theoretical OC curves shown in Figure 5.

Field data from concurrent studies. Of the 10 blocks for which data are presented in Table 2, the estimate provided by sampling scheme D is closer to the actual CTV incidence in six cases, the estimate provided by sampling scheme A is closer in two cases, and in the other two cases, no CTV-positive trees were detected by either sampling scheme.

\section{DISCUSSION}

For CTV survey programs, the main objective is to obtain estimates that are both accurate and precise, particularly at low incidence. The main constraints are the costs and labor associated

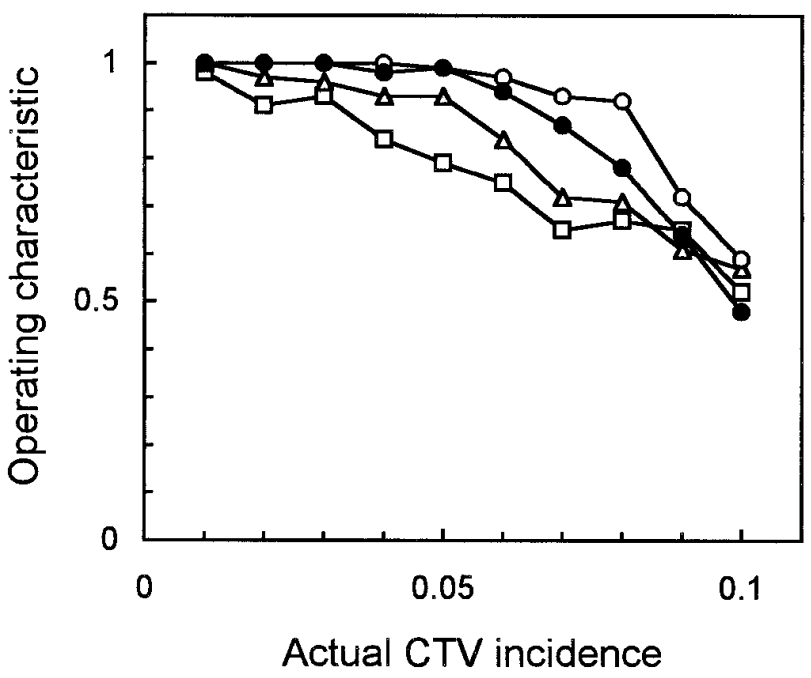

Fig. 6. Realized operating characteristic curves for each of the sampling schemes discussed in the current study: Scheme A, $-\square-$; scheme $\mathrm{B},-\Delta-$; scheme $\mathrm{C},-\mathrm{O}-$; and scheme $\mathrm{D},-\longrightarrow-$ The symbols represent the points at which values of the operating characteristic were calculated. 
with field sample collection and laboratory ELISA tests. In the current study, the results of both simulation studies and field trials show, as may be expected, that as a larger proportion of the population is sampled, there is a corresponding increase in accuracy and precision of the estimate of incidence. However, since these improvements are largely preserved when the material from groups of trees is pooled and tested without distinguishing individual trees, the proportion of the population sampled may be increased without having to increase to the same extent the number of tests required. In the current study, the groups (both actual and simulated) from which material is pooled comprised rectangular quadrats containing four trees. The benefits of the alternative sampling methodology based on this procedure are increased accuracy and precision of incidence estimates, with little additional field collection effort and only a small increase in the number of samples that undergo ELISA.

Note that the estimates that are provided by the sampling schemes discussed here (both for individual trees and for groups of trees) are not 'optimum sample size' estimates, in the sense used by Karandinos (17) and others $(14,20)$ to indicate that sample size is adjusted to obtain estimates with a fixed, prespecified level of precision. In the current study, it is the sample size that is fixed by the choice of group size and the periodicity of systematic sampling. When individuals are sampled and tested, $N$ is the total number of trees sampled (i.e., the number of 'groups' of size $n=1$ ). A measure of the realized precision of the estimate $\hat{p}_{\text {low }}$ is

$$
\operatorname{SE}\left(\hat{p}_{\text {low }}\right)=\sqrt{\hat{v}_{\text {low }} / N}
$$

in which $\hat{v}_{\text {low }}$ is obtained from equation 3. Alternatively, the calculation of $\operatorname{SE}\left(\hat{p}_{\text {low }}\right)$ may be based on $\hat{v}_{\text {low, bin }}$, obtained from equation 4 . When groups of trees are sampled and the material from each group is pooled and tested together, a measure of the realized precision of the estimate $\widetilde{p}_{\text {low }}$ can be obtained from

$$
\operatorname{SE}\left(\widetilde{p}_{\text {low }}\right)=\sqrt{\tilde{v}_{\text {low }} / N}
$$

in which $\widetilde{v}_{\text {low }}$ is obtained from

$$
\tilde{v}_{\text {low }} \approx \frac{\hat{p}_{\text {high }} \cdot\left(1-\hat{p}_{\text {high }}\right)^{(2-n) / n}}{n^{2}}
$$

derived in this format by Hughes et al. (15) and in a different format by Bhattacharyya et al. (1). Hepworth (12) and Thompson (30) have an alternative derivation. Standard errors of the estimated CTV incidence increase with increasing CTV incidence, over the range of incidence considered here. This is reflected in field data (Fig. 3), in which the scatter of points representing esti-

TABLE 2. Estimates of citrus tristeza virus (CTV) incidence made from field data

\begin{tabular}{lcccccc}
\hline & $\begin{array}{c}\text { Total } \\
\text { number } \\
\text { of trees }\end{array}$ & $\begin{array}{c}\text { Actual } \\
\text { CTV } \\
\text { incidence }^{\mathrm{b}}\end{array}$ & $\hat{p}_{\text {low }}{ }^{\mathrm{c}}$ & $\mathrm{SE}\left(\hat{p}_{\text {low }}\right)^{\mathrm{d}}$ & $\tilde{p}_{\text {low }}{ }^{\mathrm{e}}$ & $\mathrm{SE}\left(\tilde{p}_{\text {low }}\right)^{\mathrm{f}}$ \\
\hline$(07) 1-8$ & 304 & 0.003 & 0 & $\ldots$ & 0 & $\ldots$ \\
$(07) 35-46$ & 456 & 0.015 & 0 & $\ldots$ & 0.018 & 0.013 \\
$(10 \mathrm{~A}) 25-32$ & 513 & 0.019 & 0 & $\ldots$ & 0 & $\ldots$ \\
$(10 \mathrm{~B}) 34-41$ & 512 & 0.033 & 0.036 & 0.041 & 0.060 & 0.022 \\
$(11 \mathrm{~A}) 9-16$ & 512 & 0.041 & 0.036 & 0.041 & 0.040 & 0.018 \\
$(9 \mathrm{~B}) 43-50$ & 512 & 0.049 & 0.071 & 0.058 & 0.024 & 0.014 \\
$(10 \mathrm{~A}) 1-8$ & 495 & 0.065 & 0.042 & 0.046 & 0.054 & 0.022 \\
$(10 \mathrm{~B}) 42-49$ & 512 & 0.076 & 0 & $\ldots$ & 0.033 & 0.016 \\
$(9 \mathrm{~A}) 9-16$ & 512 & 0.096 & 0.143 & 0.078 & 0.111 & 0.030 \\
$(07) 47-59$ & 492 & 0.118 & 0.188 & 0.090 & 0.082 & 0.027 \\
\hline
\end{tabular}

${ }^{a}$ The blocks are arranged in order of increasing actual CTV incidence.

${ }^{b}$ Calculated from the total number of CTV-positive trees (determined by enzyme-linked immunosorbent assay) divided by the total number of trees in the block.

${ }^{\mathrm{c}}$ From equation 1 applied to data collected according to sampling scheme A.

${ }^{\mathrm{d}}$ From equation 7. SE $=$ standard error.

${ }^{\mathrm{e}}$ From equation 2 applied to data collected according to sampling scheme D.

${ }^{\mathrm{f}}$ From equation 8. mates of incidence increases with increasing incidence, and in simulated data (Fig. 4), in which the width of the intervals surrounding median CTV incidence increases with increasing incidence for all four sampling schemes.

It is clear that deciding on the group size to be used in the type of hierarchical sampling scheme proposed here is an important issue. In conventional group testing, groups are formed after sampling has taken place, and the choice of group size can, thus, be deferred until testing takes place. The choice of group size is then largely based on statistical criteria. In hierarchical sampling, the individual members of a group are in spatial proximity in the field. Because of this, the practicalities of operating the scheme in the field must be taken into account, in addition to statistical criteria. In the current study, sampling scheme D, with groups of four trees, each group arranged two by two, was selected as being appropriate for practical implementation in the field. The collection of sample material from groups comprising four immediately adjacent trees requires only moderate additional effort compared with the collection of an equivalent number of samples when the sampling unit is the individual tree.

The results of the tests of ELISA sensitivity (Table 1) demonstrated that the assay is capable of detecting CTV-positive tissue even when it is present in combination with considerable quantities of healthy tissue. As a result, it is envisaged that the collection of two leaves from each of the four trees in a group would often be appropriate for the detection of systemic infections. However, the level of virus titer does vary through the year. We therefore recommend that the type of experiment outlined above, testing the ability of ELISA to detect various mixtures of CTV-positive and CTV-negative tissue using high-quality antiserum, be repeated prior to sampling if there is doubt about the level of virus titer. We note that, currently, the CCTEA typically collects eight leaves per tree, two from each directional quadrant, in an attempt to increase the chances of detecting early, presystemic infections of CTV. We do not consider the problem of detecting presystemic infections in individual trees in this paper.

Some consideration also needs to be given to the statistical criteria for group size and, in particular, the appropriateness of a group size of four as judged by these criteria. Two issues predominate. The first is that estimates of CTV incidence made by means of equation 2 may be biased (28). The extent of the bias depends on the actual CTV incidence (the true value of $p_{\text {low }}$ ), the number of groups $(N)$, and the number of trees per group $(n)$. The prob-

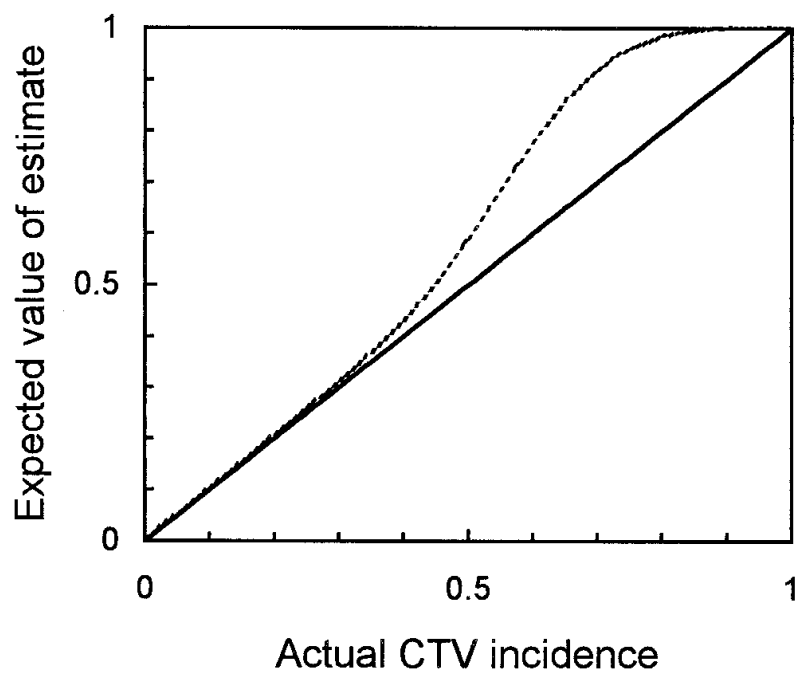

Fig. 7. The expected value of the maximum likelihood estimate $\left(\widetilde{p}_{\text {low }}\right)$ of citrus tristeza virus (CTV) incidence (the dotted line), from equation 2, is plotted against actual CTV incidence (the true value of $p_{\text {low }}$ ), with $N=25$ quadrats and $n=$ four trees per quadrat. The solid line represents an unbiased estimate. 
lem of bias tends to be more serious for small values of $N$ and large values of $n$. Using the formula provided by Swallow (28), the bias associated with estimates of CTV incidence made from sampling scheme $\mathrm{D}(N=25, n=4)$ was calculated. Figure 7 shows that the bias of such estimates is negligible up to around $p_{\text {low }}=0.4$, a range extending well beyond the range of interest in the current study.

The second issue is that of saturation. If group size is too large, all groups are likely to test positive even at small levels of $p_{\text {low }}$. It is desirable to choose a group size that will result in some groups testing positive and some negative $(4,12)$. The general conclusion, taking into account the issues of detection, bias, and saturation, is that a relatively large number of relatively small groups is preferable as a basis for group testing. This does not conflict with the additional requirement for practicability imposed by hierarchical sampling. If anything, the requirement for practicability of the method from a field collection point of view will lead to the adoption of smaller group sizes than are strictly necessary from a statistical point of view. In the case of sampling strategies for CTV assessment, if the cost of sampling were a limitation and sampling from groups larger than four were deemed a practical proposition, schemes similar to $\mathrm{D}$, but with a larger group size, could be investigated.

The use of simulation to study sampling schemes, and ultimately to validate a proposed scheme, is worthy of comment. In the context of crop protection against insect pests, plant pathogens, or weeds, the main justification for this type of approach is that it allows a sampling scheme to be tested against known standards $(18,21,31)$. In the current study, most of the standards were generated by computer simulation, but this need not be the case. Field simulation (27) and computer simulation based on field maps of diseased plants (20) are variations on the same technique. In each case, the actual level and the pattern of disease are known, and the ability of different schemes to provide estimates of disease with acceptable levels of accuracy and precision can, therefore, be compared. Validation using field data is more difficult. Extensive resources are required to provide censuses against which sampling schemes can be tested. Even so, appropriate levels of replication in the range of disease incidence that is of interest cannot be guaranteed. The main purpose served by the collection of field data during the current study (Table 2) was, therefore, the refinement of the field technique rather than the collection of a data set for a rigorous examination of the statistical properties of sampling schemes.

CTV is vectored by several aphid species $(10,11,32,33,34)$. Until recently, A. gossypii was the most common aphid vector in both U.S. and Mediterranean citrus-growing areas $(10,34)$. However, the most efficient aphid vector known worldwide, Toxoptera citricida, the brown citrus aphid $(23,33)$, was introduced into Florida in 1995 and has since spread throughout most of the citrus-producing areas of the state. T. citricida is thought to be up to 25 times more efficient at transmission of many CTV isolates compared with $A$. gossypii (33). There is also evidence to suggest that more aggregated patterns of infected trees result when T. citricida is the main vector $(9,10)$. Sampling methods for aggregated patterns of CTV infection are the subject of continuing studies.

\section{ACKNOWLEDGMENTS}

We thank the Central California Tristeza Eradication Agency (CCTEA) and the Florida Department of Agriculture and Consumer Services, Division of Plant Industry, for partial financial support for the project. We also thank K. Riley of CCTEA and P. Bell and T. Riley of USDA for technical assistance in processing samples and data and for performing serological assays.

\section{LITERATURE CITED}

1. Bhattacharyya, K. B., Karandinos, M. G., and DeFoliart, G. R. 1979. Point estimates and confidence intervals for infection rates using pooled organisms in epidemiologic studies. Am. J. Epidemiol. 109: 124-131.

2. Binns, M. R., and Nyrop, J. P. 1992. Sampling insect populations for the purpose of IPM decision making. Annu. Rev. Entomol. 37:427-453.

3. Boivin, G., Kushalappa, A. C., and Brodeur, L. 1990. Spatial dispersion and binomial sequential sampling plan for Cercospora carotae on carrots. Can. J. Plant Pathol. 12:209-212.

4. Briolini, G. 1984. Data collection and analysis in pest control. Pages 67-74 in: Statistical and Mathematical Methods in Population Dynamics and Pest Control. R. Cavalloro, ed. A. A. Balkema, Rotterdam, the Netherlands.

5. Chen, C. L., and Swallow, W. H. 1990. Using group testing to estimate a proportion, and to test the binomial model. Biometrics 46 : 1035-1046.

6. de Jong, P. D. 1995. Sampling for detection: Leek rust as an example. Int. J. Pest Manage. 41:31-35.

7. Garnsey, S. M., and Cambra, M. 1991. Enzyme-linked immunosorbant assay (ELISA) for citrus pathogens. Pages 193-216 in: Graft Transmissible Diseases of Citrus. C. N. Roistacher, ed. FAO, Rome.

8. Gibson, G. J. 1997. Investigating mechanisms of spatiotemporal epidemic spread using stochastic models. Phytopathology 87:139-146.

9. Gottwald, T. R., Cambra, M., Moreno, P., Camarasa, E., and Piquer, J. 1996. Spatial and temporal analyses of citrus tristeza virus in eastern Spain. Phytopathology 86:45-55.

10. Gottwald, T. R., Garnsey, S. M., Cambra, M., Moreno, P., Irey, M., and Borbón, J. Differential effects of Toxoptera citricida vs. Aphis gossypii on temporal increase and spatial patterns of spread of citrus tristeza. In: Proc. Conf. Int. Organ. Citrus Virol., 13th. P. Moreno, J. V. De Graça, and R. K. Yokomi, eds. Int. Organ. Citrus Virol., Riverside, CA. In press.

11. Gottwald, T. R., Garnsey, S. M., and Yokomi, R. K. 1994. Present distribution of citrus tristeza virus and its vector, the brown citrus aphid, and potential for further spread. Citrus Ind. 75:52-60.

12. Hepworth, G. 1996. Exact confidence intervals for proportions estimated by group testing. Biometrics 52:1134-1146.

13. Hughes, G., and Madden, L. V. 1992. Aggregation and incidence of disease. Plant Pathol. 41:657-660.

14. Hughes, G., Madden, L. V., and Munkvold, G. P. 1996. Cluster sampling for disease incidence data. Phytopathology 86:132-137.

15. Hughes, G., McRoberts, N., Madden, L. V., and Gottwald, T. R. 1997. Relationships between disease incidence at two levels in a spatial hierarchy. Phytopathology 87:542-550.

16. Jones, V. P. 1994. Sequential estimation and classification procedures for binomial counts. Pages 175-205 in: CRC Handbook of Sampling Methods for Arthropods in Agriculture. L. P. Pedigo and G. D. Buntin, eds. CRC Press, Boca Raton, FL.

17. Karandinos, M. G. 1976. Optimum sample size and comments on some published formulae. Bull. Entomol. Soc. Am. 22:417-421.

18. Lin, C. S., Poushinsky, G., and Mauer, M. 1979. An examination of five sampling methods under random and clustered disease distribution using simulation. Can. J. Plant Sci. 59:121-130.

19. Madden, L. V., and Hughes, G. 1995. Plant disease incidence: Distributions, heterogeneity, and temporal analysis. Annu. Rev. Phytopathol. 33: 529-564.

20. Madden, L. V., Hughes, G., and Munkvold, G. P. 1996. Plant disease incidence: Inverse sampling, sequential sampling, and confidence intervals when observed mean incidence is zero. Crop Prot. 15:621-632.

21. Nyrop, J. P., and Binns, M. B. 1990. Quantitative methods for designing and analyzing sampling programs for use in pest management. Pages 67-132 in: CRC Handbook of Pest Management in Agriculture, 2nd ed., Vol. II. D. Pimentel and A. A. Hanson, eds. CRC Press, Boca Raton, FL.

22. Perry, J. N. 1994. Sampling and applied statistics for pests and diseases. Aspects Appl. Biol. 37:1-14.

23. Rocha-Peña, M. A., Lee, R. F., Lastra, R., Niblett, C. L., OchoaCorona, F. M., Garnsey, S. M., and Yokomi, R. K. 1995. Citrus tristeza virus and its aphid vector Toxoptera citricida: Threats to citrus production in the Caribbean and Central and North America. Plant Dis. 79:437-445.

24. Rodoni, B. C., Hepworth, G., Richardson, C., and Moran, J. R. 1994. The use of a sequential batch testing procedure and ELISA to determine the incidence of five viruses in Victorian cut-flower Sim carnations. Aust. J. Agric. Res. 45:223-230.

25. Sampford, M. R. 1962. An Introduction to Sampling Theory. Oliver and Boyd, Edinburgh.

26. Seem, R. C. 1984. Disease incidence and severity relationships. Annu. Rev. Phytopathol. 22:133-150.

27. Seem, R. C., Magarey, P. A., McCloud, P. I., and Wachtel, M. F. 1985. A 
sampling procedure to detect grapevine downy mildew. Phytopathology $75: 1252-1257$

28. Swallow, W. H. 1985. Group testing for estimating infection rates and probabilities of disease transmission. Phytopathology 75:882889.

29. Swallow, W. H. 1987. Relative mean squared error and cost considerations in choosing group size for group testing to estimate infection rates and probabilities of disease transmission. Phytopathology 77:1376-1381.

30. Thompson, K. H. 1962. Estimation of the proportion of vectors in a natural population of insects. Biometrics 18:568-578.

31. Wiles, L. J., Wilkerson, L. J., and Gold, H. J. 1992. Simulating weed scouting and weed control decision making to evaluate scouting plans. Pages 1166-1171 in: Proc. Winter Simulation Conf., 1992. J. J. Swan, D.
Goldsman, R. C. Crain, and J. R. Wilson, eds.

32. Yokomi, R. K., Garnsey, S. M., Civerolo, E. L., and Gumpf, D. J. 1989. Transmission of exotic citrus tristeza virus isolates by a Florida colony of Aphis gossypii. Plant Dis. 73:552-556.

33. Yokomi, R. K., Lastra, R., Stoetzel, M. B., Damsteegt, V. D., Lee, R. F., Garnsey, S. M., Gottwald, T. R., Rocha-Peña, M. A., and Niblett, C. L. 1994. Establishment of the brown citrus aphid Toxoptera citricida (Homoptera: Aphididae) in Central America and the Caribbean Basin. J. Econ. Entomol. 88:1078-1085.

34. Yokomi, R. K., and Oldfield, G. N. 1991. Seasonal fluctuations of alate aphid activity in California citrus groves. Pages 71-76 in: Proc. Conf. Int. Organ. Citrus Virol., 11th. R. H. Brlansky, R. F. Lee, and L. W. Timmer, eds. Int. Organ. Citrus Virol., Riverside, CA. 